\title{
Left main stenting induced flow disturbances on ascending aorta and aortic arch
}

\author{
Gianluca Rigatelli ${ }^{1}$, Marco Zuin ${ }^{1,2}$, Alan Fong ${ }^{3}$, Truyen TTT Tai ${ }^{4}$, Thach Nguyen ${ }^{4}$ \\ 'Cardiovascular Diagnosis and Endoluminal Interventions Unit, Rovigo General Hospital, Rovigo, Italy; \\ ${ }^{2}$ Section of Internal and Cardiopulmonary Medicine, University of Ferrara, Ferrara, Italy; \\ ${ }^{3}$ Department of Cardiology, Clinical Research Center, Sarawak General Hospital, Sarawak, Malaysia; \\ ${ }^{4}$ Director of Cardiovascular Research, Methodist Hospital, Merrillville, IN 46410, USA; Tan Tao University \\ School of Medicine, Long An, Vietnam
}

\section{ABSTRACT}

Background and Objectives: Ostial LM stenting potentially induces turbulence in the aortic wall near the LM ostium, which might be correlated with aorta dilation and dissection. We investigated through a computational fluid dynamic analysis (CFD), the presence and potential consequences of flow turbulences both in the ascending aorta and arch after a stenting left main (LM) mid shaft or distal disease. Methods: The model of the ascending aorta and left coronary artery was reconstructed reviewing both angiographic and echocardiographic measurements of 80 consecutive patients ( 43 males, mean age $75.1 \pm 6.2$ years) with significant LM mid shaft or distal disease treated in our institution. For stent simulation, a third-generation everolimuseluting stent was reconstructed. Two stenting procedures (lesion 1:1 or ostial coverage) were investigated. Results: The net area averaged WSS of the model resulted higher when the stent covered the lesion 1:1 compared to the ostial coverage (3.68 vs. $2.06 \mathrm{~Pa}, P=0.01$ and 3.97 vs. $1.98 \mathrm{~Pa}, P<0.001$, respectively). LM ostial coverage generates more turbulences in the LM itself, in the aortic wall at ostium level, and at the sino-tubular junction compared with the stenting of the lesion 1:1. Conversely, in the ascending aorta, the WSS appears lower when stenting the lesion 1:1. Conclusions: Extending the stent coverage up to the ostium, when the ostial region is not diseased, might induce unfavorable alterations of flow; not only both at the level of the LM lesion and ostium sites, but also in the ascending aorta and aortic arch, potentially predisposing the aortic wall to long-term damage.

Key words: coronary stent, left main, physiology, interventional, computed fluid dynamic

\section{INTRODUCTION}

Effects of coronary stenting techniques are generally investigated regarding wall shear stress (WSS) or turbulence of flow at the lesion or bifurcation sites. ${ }^{[1-2]}$ Recently, we speculated that ostial left main (LM) stenting potentially induces turbulence in the aortic wall near to the LM ostium. ${ }^{[3]}$ Potential impact on the ascending aorta and arch have never been evaluated despite the fact that it would be logical that any turbulence induced outside the coronary tree can propagate at a certain distance within the aortic vasculature. Noticeably, higher WSS within the ascending aorta have been suspected to be involved in aortic aneurysm and dissection. ${ }^{[4-5]}$ The aim of our study is to investigate, by means of computation fluid dynamic analysis (CFD), the presence and potential impact of turbulences induced by ostial LM stenting, not only in the LM but also within the ascending aorta and arch.

\section{METHODS}

\section{Construction of the virtual model}

For the computational domain analysis, we reconstructed the ascending aortic arch from the plane of the aortic valve to the left subclavian artery. Both coronary ostia were also modelled, with particular attention to the left coronary stem. The model of ascending aorta and arch and LM was based 
on the dimensions obtained after analyzing transthoracic echocardiography (TTE) and coronary angiography (CA) of 80 consecutive patients (mean age $75.1 \pm 6.2$ years, 43 males) with significant $(>50 \%$ ) luminal narrowing on quantitative coronary analysis (QCA) LM mid shaft or distal disease. These patients had no significant ascending aorta or aortic valve disease, and they had undergone percutaneous coronary intervention (PCI) between January 1 $1^{\text {st }}, 2015$ and January $1^{\text {st }}, 2017$. The mean diameter of proximal LM, left anterior descending artery (LAD), and left circumflex (LCX), evaluated with QCA were $4.32 \pm 0.82 \mathrm{~mm}, 3.21 \pm 0.65 \mathrm{~mm}$, and $2.52 \pm 0.88 \mathrm{~mm}$, respectively. The mean LAD-LCX bifurcation angle, measured after the diagnostic angiography using an electronic goniometer was $52.6 \pm 10.4^{\circ}$, whereas the mean length of the $\mathrm{LM}$ was $18.5 \pm 2.7 \mathrm{~mm}$. Mean diameter stenosis by QCA was $88 \pm 7.6 \%$, while mean ascending aorta diameter measured, as previously suggested ${ }^{[6]}$ at the sinotubular region and before the emergence of right anonymous trunk, was $28.8 \pm 7.9 \mathrm{~mm}$ and $28.2 \pm 6.7 \mathrm{~mm}$, respectively. Following these measurements, the diameters of LCA and LCX were modelled as following: LM $4.5 \mathrm{~mm}$, LAD $3.5 \mathrm{~mm}$ and LCX $2.75 \mathrm{~mm}$, with bifurcation angle set up at $55^{\circ}$. A $12 \mathrm{~mm}$ length plaque inducing a stenosis of $90 \%$ was placed at the mid-shaft position (Figure 1). The height of the aortic take off (distance between the origin of the LM and the aortic valve plane) was set at $10 \mathrm{~mm}$, while the diameter of ascending aorta just above the left coronary origin was set at $28 \mathrm{~mm}$ (Figure 2). The model was constructed using Rhinoceros v. 4.0 Evaluation (McNeel \& Associates, Indianapolis, IN). The systemic pressure was assumed to be stable at $120 / 80 \mathrm{mmHg}$.

\section{Stent geometry reconstruction}

For the stent simulation, we reconstructed the strut design and linkage pattern of a third-generation, everolimus-eluting stent (ORSIRO stent, Biotronik IC, Bulack, Switzerland), commonly used in our institution. The strut thickness of this stent is characterized by a very ultrathin strut $(60 \mu \mathrm{m}$ up to $3.0 \mathrm{~mm}$ diameter stent and $80 \mu \mathrm{m}$ up to $4.0 \mathrm{~mm}$ stent, Figure 2). Computer Aided Design (CAD) software was used to reproduce the stented geometry as accurately as possible (SolidWorks 2009, Solidworks Corp, Concord, MA). In the first step, we created the solid model of the coronary artery bifurcation and then the expanded stent geometry. For this purpose, a hollow tube with outer diameter equal with both the nominal expanded diameter and thickness of the stent was created. Then, a 2-dimensional sketch with the stent strut was propagated and wrapped around the tube. Through a cut-out, the obtained ring of the stent was propagated axially to create the full-length expanded model.

\section{Virtual implantation}

After placing the stent model in the correct position, the stenting procedure was performed following the real procedural steps in the following manner: lesion only or extending the stent coverage up to the ostium in order to assess the potential impact on ascending aorta fluid dynamic.

\section{Lesion 1:1:}

- Predilation with non-compliant Euphora (Medtronic Inc, USA) balloon $3.0 \times 12 \mathrm{~mm}$ at $16 \mathrm{~atm}$

- Stent implantation: Orsiro $4.0 \times 12 \mathrm{~mm}$ at 18 atm covering the lesion 1:1

- Over-dilation with $4.5 \times 12 \mathrm{~mm}$ non-compliant Euphora (Medtronic Inc, USA) balloon at $20 \mathrm{~atm}$
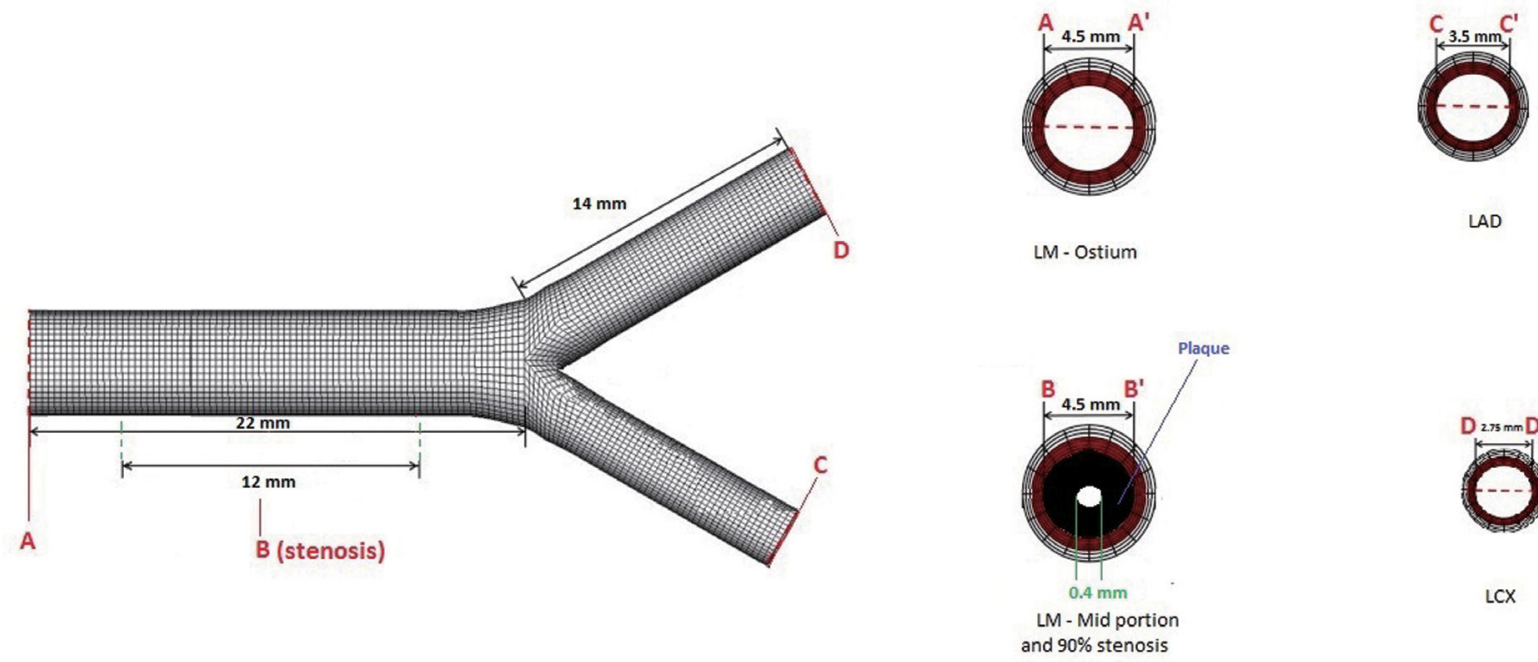

LAD

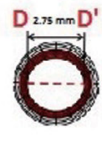

LCX

Figure 1: Left Coronary stem model details: diameters of the left circumflex and left anterior descending coronary artery were calculated following the Finet Law. 


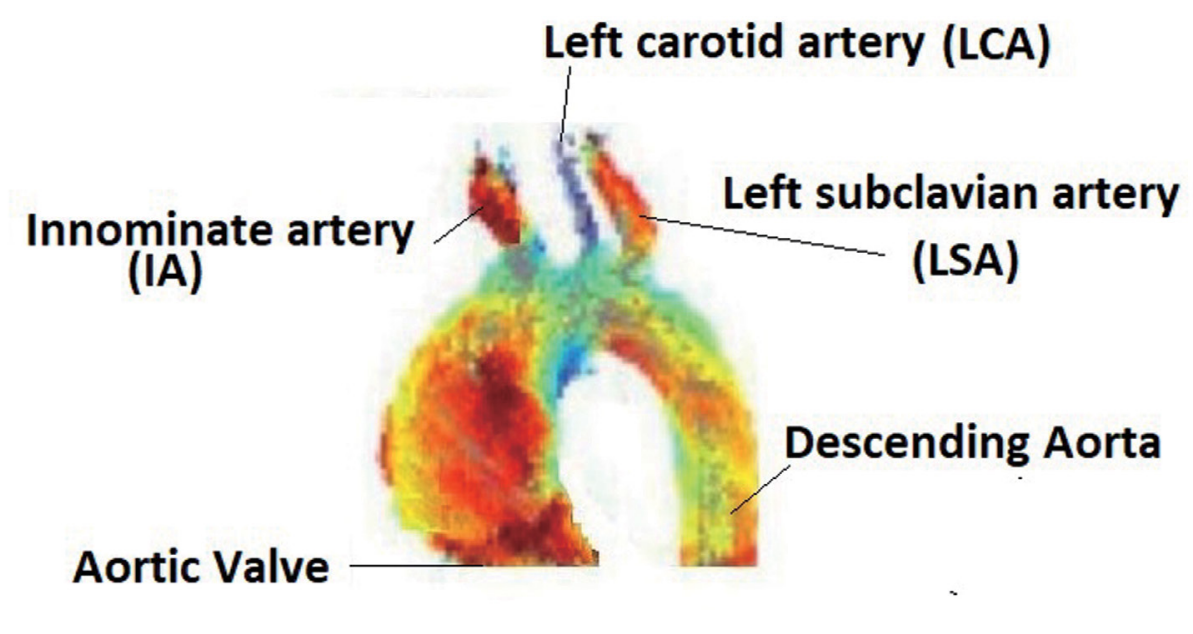

WSS (Pa)

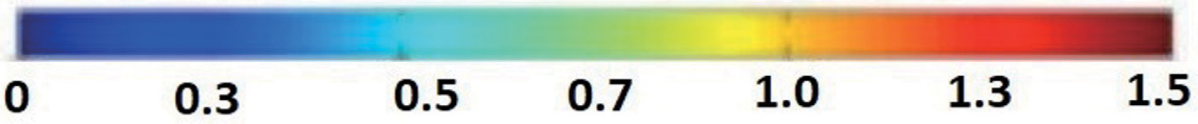

Figure 2: Computational fluid dynamic of the ascending aorta and aortic arch model at basal conditions before any stenting procedure.

\section{Ostial coverage:}

- Predilation with non-compliant Euphora (Medtronic Inc, USA) balloon $3.0 \times 12 \mathrm{~mm}$ at $16 \mathrm{~atm}$

- Stent implantation: Orsiro $4.0 \times 15 \mathrm{~mm}$ at $18 \mathrm{~atm}$ placed from the lesion itself up to the ostium with no protrusion into the aorta

- Over-dilation with $4.5 \times 15 \mathrm{~mm}$ non-compliant Euphora (Medtronic Inc, USA) balloon at 20 atm

Using the Boolean operation, the modified solid model was subtracted from the bifurcation model to obtain the final geometry. We assumed that after stent deployment and implantation, there was no residual stenosis.

\section{Computational fluid dynamic analysis}

Blood was modelled as a non-Newtonian, viscous and incompressible fluid. Density was defined as $1060 \mathrm{~kg} / \mathrm{m}^{3}$, according to the standard values cited in the literature. ${ }^{[7-10]}$ Blood was represented by the Navier-Stokes and continuity equations.

Instead, the Carreau model was applied for the viscosity of blood. Considering that coronary perfusion is mainly diastolic, we performed a steady flow simulation using a basal diastolic pressure of $80 \mathrm{mmHg}(10665 \mathrm{~Pa})$. In the analysis, the static pressure (Pa), wall shear stress (WSS) $\mathrm{Pa}$ and Reynolds number were evaluated at the lesion site and ostium, whereas the velocity streamlines and the wall shear stress (WSS) (Pa) were evaluated along the ascending aorta and arch.

Static pressure in the vessel was evaluated in Pascal. From a pathophysiological point of view, low static pressure is generally related to increased vessel wall thickness.

WSS was defined as the force that is tangentially acting on the surface due to friction. As it is well known that low WSS are related to the development of greater plaque, higher neo-endothelization and necrotic core progression with a constrictive remodeling, whereas high WSS segments develop greater necrotic core and calcium progression with expansive remodeling. ${ }^{[1]]}$ The numeric grid was obtained with ANSYS Meshing 14.0 (Ansys, Inc., Canonsburg, PA), while the simulations were conducted using the commercial software ANSYS FLUENT 14.0 (Ansys, Inc., Canonsburg, $\mathrm{PA})$.

\section{Statistical analysis}

Continuous variables were expressed as mean and standard deviation (SD) and compared with the Student's t-test, while differences of WSS were evaluated as delta between the mean WSS values at the same levels in the two models. All 
statistical analyses were carried out using the SPSS statistical software version 19.0 (SPSS Inc, Chicago, IL, USA). A $P$ value $<0.05$ was considered statistically significant.

\section{RESULTS}

The net area averaged WSS of the model resulted higher when the stent covered the lesion 1:1 compared to the ostial coverage (3.68 vs. $2.06 \mathrm{~Pa}, P=0.01$ and 3.97 vs. 1.98 $\mathrm{Pa}, P<0.001$, respectively). Similarly, the static pressure and the Reynolds number were significantly higher after the stent coverage of the ostium as shown in Table 1. LM stenting produced alterations of the fluid dynamic into the ascending aorta and arch. As a matter of fact, the LM ostial coverage induced more turbulence in the LM itself and in the aortic wall at the ostium level and at the sinotubular junction (Figure 3) compared with the stenting of lesion 1:1. In the ascending aorta, the WSS appeared to be lower when stenting lesion 1:1 but only at site 1 (Figure 4), whereas at site 2 , the differences between WSS and areas of low WSS were not statistically significant between stenting up to the ostium and lesion 1:1 (Table 2). The differences in WSS were $0.8 \pm 0.1 \mathrm{~Pa}$ at site 1 and $0.1 \pm 0.2 \mathrm{~Pa}$ at site 2 , with a difference in mean area average of $0.31 \pm 0.3 \mathrm{~Pa}$.

\section{DISCUSSION}

Our computational simulation suggested that LM stenting with ostial coverage, different from stenting only on the mid-shaft lesion, induced flow alteration not only at lesion and ostium sites but also in the ascending aorta and aortic arch regions, potentially causing an increase of WSS.

Stenting of LM requires a different strategy depending on whether the location of the lesion is the ostium, mid shaft, distal or bifurcation. While percutaneous treatment of left main bifurcation disease is still problematic, stenting of ostial and mid-shaft disease is currently widely accepted as a safe alternative to surgery. When mid shaft lesions or distal lesions are to be treated, to extend the strut coverage up to the ostium, or to stent the lesion only is a matter of the operators' choice. Our study suggested that there are some reasons for preferential stenting of a lesion 1:1 over extending the stent up to the ostium.

As a matter of fact, in our study, at the lesion site, to extend the lesion coverage up to the ostium resulted in a decrease in WSS and increase in hydrostatic pressure and the Reynolds number of the models, potentially increasing the chance of restenosis and thrombosis, as suggested by LaDisa et al. ${ }^{[12]}$ Seo et al.,$^{[13]}$ and Koskinas et al..$^{14]}$ On the contrary, at the ostium site, a high WSS caused an increase of flow turbulence, and similarly, into the ascending aorta and aortic arch compared to the stenting the lesion on a 1:1 fashion. A propagation to the ascending aorta seems the be an intuitive mechanism, and also, the straightness of the aortic wall section at the ostium caused by ostial stenting might contribute to regional increase of rigidity with subsequent increase in WSS.

As matter of fact, stent implantation at any site induces a flow separation and recirculation immediately downstream of the stents. In a steady flow, within straight vessels, the extent of flow disturbance downstream of the stent increases with both the Reynolds number and the stent wire thickness, but is relatively insensitive to stent interwire spacing. ${ }^{[12]}$ These flow disturbances are probably caused by the stent properties and by vessel straightening the stent that conforms to the natural curvature of the artery and causes a reduction in the radius of curvature and subsequent increase in the stiffness

\begin{tabular}{|c|c|c|c|c|}
\hline & Baseline & Lesion 1:1 & Ostial coverage & $P$ \\
\hline \multicolumn{5}{|l|}{ WSS } \\
\hline Mean WSS [Pa] & 6.31 & 3.21 & 2.30 & 0.01 \\
\hline Mean area averaged WSS of the model $[\mathrm{Pa}]$ & 6.33 & 3.70 & 2.12 & 0.01 \\
\hline \multicolumn{5}{|l|}{ Static Pressure } \\
\hline Static pressure $[\mathrm{Pa}]$ & 1060.25 & 10680.35 & 10740.24 & 0.01 \\
\hline Mean area averaged Static pressure of the model $[\mathrm{Pa}]$ & 1052.20 & 1068.91 & 1090.52 & 0.01 \\
\hline \multicolumn{5}{|l|}{ Reynolds Number } \\
\hline Reynolds number & 3.30 & 6.160 & 2.980 & $<0.001$ \\
\hline Mean area averaged Reynolds Number of the model & 3.30 & 6.230 & 10.630 & 0.01 \\
\hline
\end{tabular}

Table 2: Evaluation of WSS within the ascending aorta and arch

\begin{tabular}{llll}
\hline \multicolumn{1}{l}{ Lesion only } & Ostial coverage & $P$ \\
\hline WSS & & & \\
\hline Mean WSS at site $1[\mathrm{~Pa}]$ & $0.4 \pm 0.3$ & $1.20 \pm 0.2$ & $<0.01$ \\
Mean WSS at site 2 $[\mathrm{Pa}]$ & $1.2 \pm 1.1$ & $1.3 \pm 0.9$ & 0.89 \\
Mean area averaged WSS at site $1[\mathrm{~Pa}]$ & $0.98 \pm 0.4$ & $1.29 \pm 0.6$ & 0.58 \\
\hline
\end{tabular}




\section{Ostial stenting}
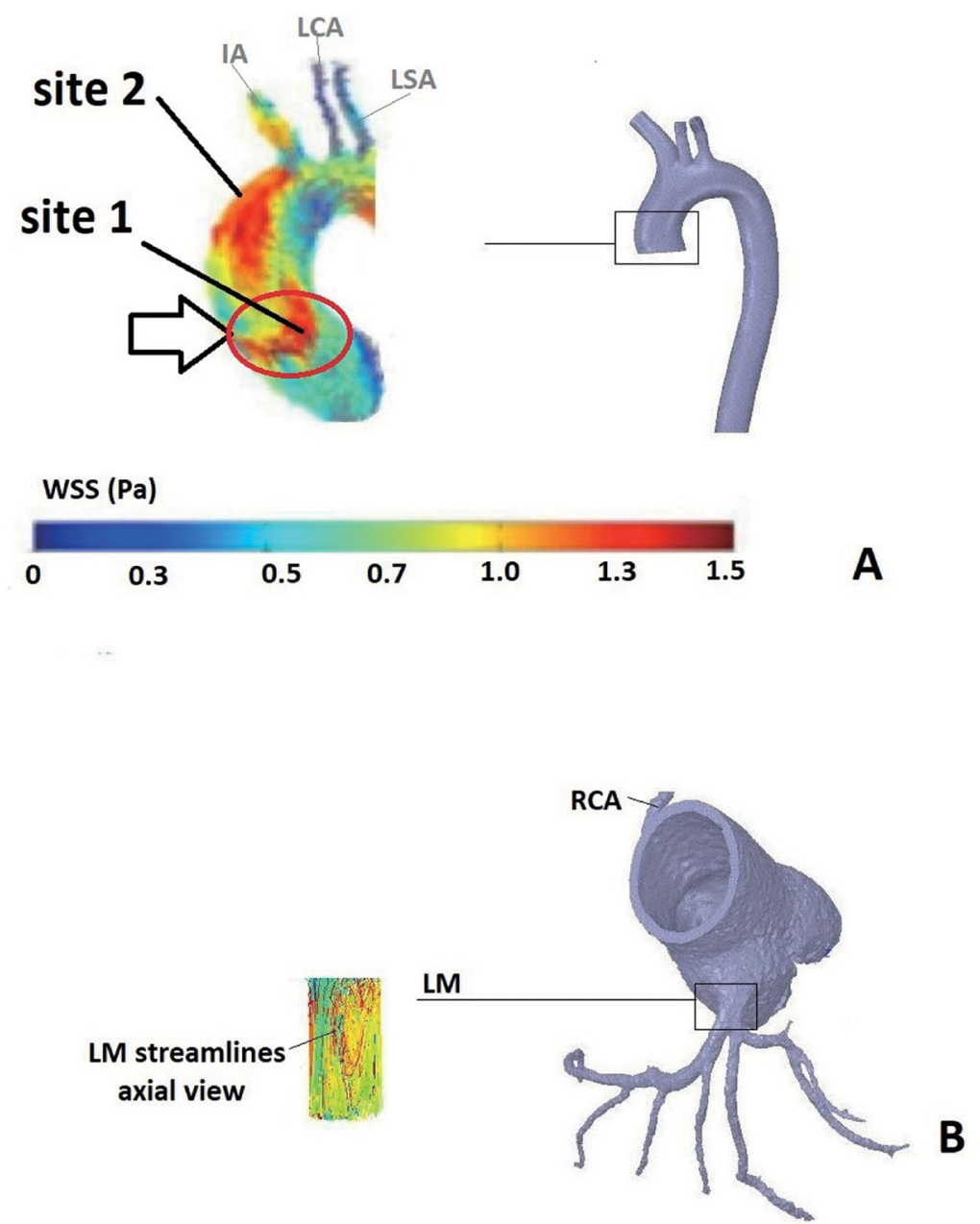

Figure 3: Computational fluid dynamic of the ascending aorta and aortic arch model after stenting of the Left Main with extension of the stent to the ostium. (A) WSS are severely regionally increased at the sino-tubular junction. (B)Similarly Left Main streamlines showed an increase of flow turbulences.

within the stented region. This straightening leads to modest skewing of the velocity profile at the inlet and outlet of the stented region where alterations in indices of WSS are most pronounced. ${ }^{[15]}$

Intuitively, the placement of a rigid stent within an elastic vessel produces wave reflection sites at the entrance to and exit from the stent. The net hemodynamic effects of these reflections depend critically on the degree of stiffness of the stent and on its length and position within the diseased vessel. In LM, stenting up to the ostium might result in an increase of rigidity also in the region of the ascending aorta near to the ostium.

Alderdson et al. showed that the presence of the stent within the larger diseased vessel has the effect of producing higher pressure at the vessel entrance than that at exit. ${ }^{[16]}$ This pressure difference, when superimposed on the underlying pressure distribution within the vessel, has the net effect of actually aiding rather than impeding the flow, but the extent of this depends on the length and position of the stent. Similar to the situation where LM is stented in the mild shaft only, a short stent placed near the entrance of the diseased vessel may be favored clinically for producing the least perturbation in the underlying hemodynamics, possibly avoiding an increase of aortic wall rigidity near the LM ostium.

Propagation of flow disturbances and increased WSS in the ascending aorta and aortic arch do not have the same effect of increasing WSS in any coronary vessel. In the latter, the high WSS seem to offer a protection against restenosis 


\section{Lesion stenting}
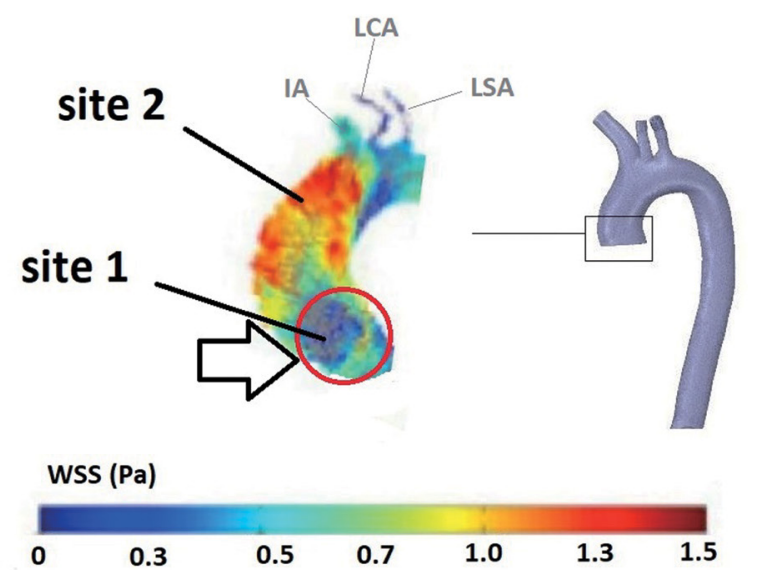

\section{A}

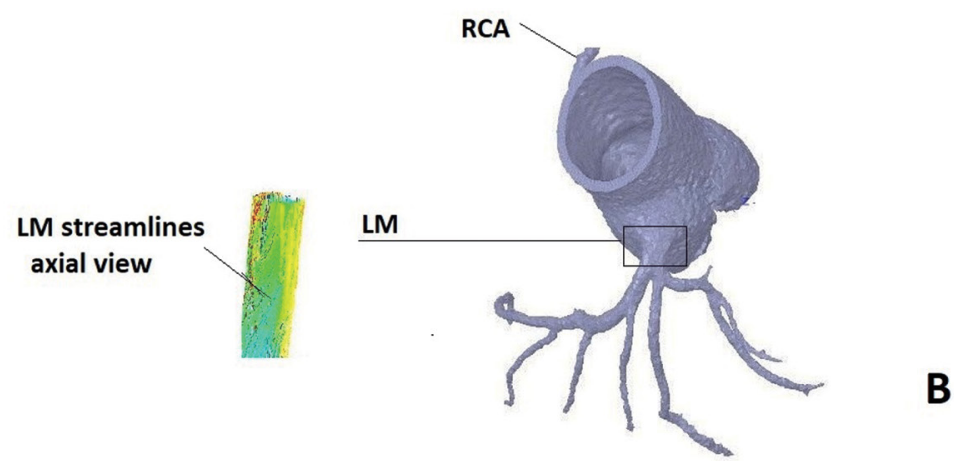

Figure 4: Computational fluid dynamic of the ascending aorta and aortic arch model after stenting of the Left Main mid shaft lesion 1:1. (A) At sino-tubular junction WSS are much lower compared to stenting with ostium coverage. (B) Left Main streamlines showed a much less turbulent flow compared to stenting with ostium coverage.

and thrombosis; while in the former, the increased WSS recently have been correlated to propagation of retrograde aortic type $\mathrm{B}$ dissection ${ }^{[17]}$ and the onset of aortic type A dissection. ${ }^{[4]}$

The increase of turbulence and flow alterations are well known phenomena associated with enlargement of aortic aneurysms, ${ }^{[18]}$ and in general, it appears clear that an increase in the turbulence in the ascending aorta and aortic arch might cause damage of the aortic endothelium, leading to both dilation and atherosclerotic plaques, and thus, should be avoided. Dilation of the ascending aorta and alterations in the branching angles seem to be the key determinants of a high WSS and aortic dissection. ${ }^{[19]}$ This recent evidence reinforced the suggestion to avoid stent extension to cover up the ostium of the LM when the ostium is not significantly diseased.

\section{Limitations}

Our study considers virtual LM and ascending aorta and arch models. The vessels have been considered noncompliant, straight and with a steady diastolic blood flow in a virtual hemodynamically stable patient. However, coronary artery perfusion is mainly diastolic and previous studies have already demonstrated that myocardial motion has a negligible effect on blood flow distribution on the coronary tree. Our model considered an optimal stent deployment without residual stenosis despite the fact that in daily clinical practice, the different angles, the amount and circumferential extent of the calcium, the length of the respective lesion and many other parameters have an obvious impact on the implantation technique and outcomes. Other limitations of the study are that we did not evaluate the time averaged WSS, oscillatory index and the relative residence time, which had a recognized role in the development of arterial atherosclerosis. 


\section{CONCLUSIONS}

Our study confirmed the previous findings, and suggests that to a certain extent, stent coverage up to the ostium when the ostial region is not diseased might induce unfavorable alterations of blood flow not only at the LM lesion and ostium sites but also in the ascending aorta and aortic arch, potentially exposing the aortic wall to longterm damage. Further studies should be undertaken in order to establish if these findings translate into a clinical disadvantage.

\section{Conflicts of Interest}

None declared

\section{REFERENCES}

1. Katritsis DG, Theodorakakos A, Pantos I, Gavaises M, Karcanias N, Efstathopoulos EP. Flow patterns at stented coronary bifurcations: computational fluid dynamics analysis. Circ Cardiovasc Interv 2012; 5: 530-9.

2. Rigatelli G, Zuin M, Dell'Avvocata F, Vassilev D, Daggubati R, Nguyen $\mathrm{T}, \mathrm{V}$, et al. Evaluation of coronary flow conditions in complex coronary artery bifurcations stenting using computational fluid dynamics: Impact of final proximal optimization technique on different double-stent techniques. Cardiovasc Revasc Med 2017; 18: 233-40

3. Rigatelli G, Zuin M, Nguyen T. Rheolytic Effects of Left Main Mid-Shaft Stenting: A Computational Flow Dynamic analysis. Adv Cardiovasc Ther in press.

4. Chi Q, He Y, Luan Y, Qin K, Mu L. Numerical analysis of wall shear stress in ascending aorta before tearing in type A aortic dissection. Comput Biol Med 2017; 89: 236-47.

5. Saikrishnan N, Mirabella L, Yoganathan AP. Bicuspid aortic valves are associated with increased wall and turbulence shear stress levels compared to trileaflet aortic valves. Biomech Model Mechanobiol 2015; 14: 577-88.

6. Evangelista A, Flachskampf FA, Erbel R, Antonini-Canterin F, Vlachopoulos $\mathrm{C}$, Rocchi G, et al. Echocardiography in aortic diseases: EAE recommendations for clinical practice. Eur J Echocardiogr 2010; 11: 645-58.

7. Cho YI, Kensey KR. Effects of the non-Newtonian viscosity of blood on flows in a diseased arterial vessel. Part 1: steady flows. Biorheology 1991; 8: 241-62.
8. Johnston BM, Johnston PR, Corney S, Kilpatrick D. Non-Newtonian blood flow in human right coronary arteries: steady state simulations. J Biomech 2004; 37: 709-20.

9. Theodorakakos A, Gavaises M, Andriotis A, Zifan A, Liatsis P, Pantos I, et al. Simulation of cardiac motion on non-Newtonian, pulsating flow development in the human left anterior descending coronary artery. Phys Med Biol 2008; 53: 4875-9.

10. Nichols WW, O'Rourke MF. The nature of flow of a liquid.In: McDonald's Blood Flow in Arteries: Theoretical, Experimental and Clinical Principles. 4th ed. London, UK: Arnold; 1998: 11-53.

11. Karino T, Goldsmith HL, Motomiya M, Mabuchi S, Sohara Y. Flow patterns in vessels of simple and complex geometries. Ann N Y Acad Sci 1987; 516: 422-41.

12. LaDisa JF Jr, Olson LE, Molthen RC, Hettrick DA, Pratt PF, Hardel MD, et al. Alterations in wall shear stress predict sites of neointimal hyperplasia after stent implantation in rabbit iliac arteries. Am J Physiol Heart Circ Physiol 2005; 288: H2465-75.

13. Seo T, Schachter LG, Barakat AI. Computational study of fluid mechanical disturbance induced by endovascular stents. Ann Biomed Eng 2005; 33: 444-56.

14. Koskinas KC, Chatzizisis YS, Antoniadis AP, Giannoglou GD. Role of Endothelial Shear Stress in Stent Restenosis and Thrombosis : Pathophysiologic Mechanisms and Implications for Clinical Translation. JACC 2012; 59: 1337-49.

15. LaDisa JF Jr, Olson LE, Douglas HA, Warltier DC, Kersten JR, Pagel PS. Alterations in regional vascular geometry produced by theoretical stent implantation influence distributions of wall shear stress: analysis of a curved coronary artery using 3D computational fluid dynamics modeling. Biomed Eng Online 2006; 5: 40.

16. Alderson $\mathrm{H}$, Zamir M. Effects of stent stiffness on local haemodynamics with particular reference to wave reflections. J Biomech 2004; 37: 339-48.

17. Osswald A, Karmonik C, Anderson JR, Rengier F, Karck M, Engelke J, et al. Elevated Wall Shear Stress in Aortic Type B Dissection May Relate to Retrograde Aortic Type A Dissection: A Computational Fluid Dynamics Pilot Study. Eur J VascEndovasc Surg 2017; 54: 324-30.

18. Khanafer KM, Bull JL, Upchurch GR Jr, Berguer R. Turbulence significantly increases pressure and fluid shear stress in an aortic aneurysm model under resting and exercise flow conditions. Ann Vasc Surg 2007; 21: 67-74.

19. Neofytou P, Tsangaris S, Kyriakidis M. Vascular wall flow-induced forces in a progressively enlarged aneurysm model. Comput Methods Biomech Biomed Engin 2008; 11: 615-26.

How to cite this article: Rigatelli G, Zuin M, Fong A, Tai TT, Nguyen T. Left main stenting induced flow disturbances on ascending aorta and aortic arch. J Transl Int Med 2019; 7: 22-8. 